\title{
Low-Rank Matrix Denoising Algorithm-Based Magnetic Resonance Imaging Combined with Computed Tomography Images in the Diagnosis of Cerebral Aneurysm
}

\author{
Daigui Zhang $\mathbb{D}^{1},{ }^{1}$ Lihua Zhou $\mathbb{D}^{1},{ }^{1}$ Tingdi Zhang $\mathbb{D}^{2}$, Shuai Wang $\mathbb{D}^{1}$, and Yue Li $\mathbb{D}^{1}$ \\ ${ }^{1}$ Department of Radiology, Jingmen No. 1 People's Hospital, Jingmen 448000, Hubei, China \\ ${ }^{2}$ Hubei University of Medicine, Shiyan 448000, Hubei, China \\ Correspondence should be addressed to Yue Li; 15610803110587@post.eurasia.edu
}

Received 12 May 2021; Accepted 26 June 2021; Published 6 July 2021

Academic Editor: Gustavo Ramirez

Copyright ( 12021 Daigui Zhang et al. This is an open access article distributed under the Creative Commons Attribution License, which permits unrestricted use, distribution, and reproduction in any medium, provided the original work is properly cited.

\begin{abstract}
This study was to analyze the diagnostic effects of computed tomography (CT) and magnetic resonance imaging (MRI) in patients with cerebrovascular diseases (CVDs) based on low-rank matrix denoising (LRMD) algorithm. The LRMD algorithm was adopted for MRI diagnosis and CT diagnosis for comparative analysis. 129 CVD patients were selected as the research objects, 43 cases were diagnosed by CT, 43 cases were diagnosed by MRI under LRMD, and the other 43 cases were diagnosed by CT + MRI. The results showed that the diagnostic compliance rates (DCRs) of CT group in the cerebral hemorrhage $(\mathrm{CH})$, cerebral infarction (CI), and cerebral aneurysm (CA) were $95.1 \%, 94.7 \%$, and $70 \%$, respectively, while those in the MRI group were $99.01 \%, 97.71 \%$, and $100 \%$, respectively. Thus, it was obtained that MRI diagnosis was much better than CT diagnosis, and CT + MRI showed the best diagnosis efficacy, showing statistical differences $(P<0.05)$. The accuracy, sensitivity, and specificity of MRI diagnosis under the LRMD algorithm were $96.28 \%, 88.76 \%$, and $90.62 \%$, respectively, which were superior to those of CT diagnosis $(92.71 \%, 84.94 \%$, and $80.71 \%$, respectively). The diagnosis cost per case (DC/C) $(799.73 \pm 100.02$ yuan) and the total diagnosis cost (TDC) $(58,521.67 \pm 301.62$ yuan) in the MRI group were higher than those in the CT group (601.42 \pm 83.61 yuan and 39,819.2 \pm 198.72 , respectively) $(P<0.05)$. In conclusion, CT + MRI under the LRMD algorithm showed good potential in diagnosis of CVD; MRI based on the LRMD algorithm showed a higher positive rate in the diagnosis of CA and was better than CT diagnosis, and $\mathrm{CT}+\mathrm{MRI}$ showed the best diagnosis effect and could improve the clinical diagnosis rate.
\end{abstract}

\section{Introduction}

Cerebrovascular disease (CVD) is a brain disease with extremely high disability and fatality rate, and cerebral aneurysm (CA) is the main cause. The main clinical manifestations are cystic protrusions on the walls of cerebral blood vessels [1]. CVD has a risk of morbidity at all ages, and the incidence is higher in middle-aged and elderly people [2]. According to the statistics of the health organization, the incidence of intracranial aneurysm rupture accounts for more than $80 \%$ each year. Intracranial aneurysm rupture and bleeding are the main causes of death and disability. The main symptoms are mostly caused by bleeding, and some are caused by tumor compression, arterial spasm, or embolism [3-5]. It is now generally believed that the risk of CA is mainly related to the destruction of the arterial wall. Hypertension, arteriosclerosis, age, smoking, and other factors can cause the rupture of the cerebral artery wall, but the cause of CA is still being studied [6]. CA has a large number of macrophages, monocytes, and $\mathrm{T}$ lymphocytes infiltrated. Studies have suggested that such inflammatory cells are closely related to the occurrence and development of CA $[7,8]$. The influence of vascular hemodynamic force causes the vascular wall to bulge, which leads to the formation, development, and rupture of CA $[9,10]$. Based on this, timely detection and diagnosis of CA are essential.

The most commonly used method for diagnosing CA is $\mathrm{X}$-ray examination. Although the X-ray examination has a good diagnostic effect, it also has certain limitations. It is prone to misdiagnosis and missed diagnosis [11]. It will affect the patient's later treatment and disease prevention [12]. 
With the continuous development of clinical diagnosis technology, computed tomography (CT) diagnosis and magnetic resonance imaging (MRI) diagnosis have been more and more used, and they also have a more accurate diagnosis effect for the diagnosis of cerebrovascular [13]. Image denoising is a common matter in image processing, so the size of the solution space needs to be limited so as to obtain a true solution $[14,15]$. Introducing image priors into the model can obtain corresponding positive terms to limit the solution space of the image [16]. The low-rank matrix denoising (LRMD) algorithm has become a research hotspot in recent years due to its better denoising performance. However, with the increase in noise intensity, the noise greatly destroys the low rank of the image so that insufficient denoising is found [17]. The prior information of the MRI image was incorporated into the LRMD model to improve the denoising effect in this study so that the details of the image itself were preserved to the greatest extent while removing the noise.

In summary, the LRMD algorithm was adopted to optimize the denoising of CT images and MRI images and applied to the clinical diagnosis of CVD patients in this study. The diagnostic effects of CT images and MRI images optimized by the LRMD algorithm were tested to evaluate the image optimization ability of the algorithm and to measure the application value of the algorithm in the diagnosis of CT images and MRI images of clinical CVD patients.

\section{Materials and Methods}

2.1. Selection and Grouping of Research Objects. 129 pathologically confirmed CVD patients who came to hospital from December 2018 to December 2020 were selected as the research objects, including 95 males and 34 females, aged 10 65 years (with an average age of $48 \pm 3.51$ years). They were rolled into a CT group, a MRI group, and a CT + MRI group according to different diagnostic methods, with 43 cases in each group. The experiment had been approved by the Medical Ethics Committee of hospital, and the patients and their families had understood and signed the informed consent forms.

The inclusion criteria were defined as follows: patients with varying degrees of nausea, vomiting, headache, and other symptoms when overworked or emotionally excited; patients who were informed and signed the surgical consent form voluntarily; and patients with clear consciousness and being able to cooperate with the experiment.

The exclusion criteria were defined as follows: patients with other malignant tumors; patients who withdrew from the experiment due to their own reasons; patients with severe dysfunction of other organs and unable to undergo surgery; patient who suffered with surgical contraindications; and patient who had speech and communication impairment, hearing impairment, or severe cognitive impairment.

\subsection{CT and MRI Examinations}

CT Diagnosis. 43 patients were diagnosed with the SOMATOM Definition AS128-slice spiral CT produced by the German Siemens. The nonionic contrast medium was used for imaging, and the part between the second cervical vertebra and the top of the skull and retrograde was scanned back and forth. The suspicious parts and cases were performed with the enhanced CT scan. The specific parameters for scanning were determined as follows: layer thickness and layer spacing were $7 \mathrm{~mm}$, tube current was about $600 \mathrm{~mA}$, tube voltage was $120 \mathrm{kV}$, scanning field of view was $25 \mathrm{~cm}$, display field was $15 \sim 25 \mathrm{~cm}$, and the collimation was set to $20 \mathrm{~m}$.

MRI Diagnosis. 43 patients were diagnosed using the $1.5 \mathrm{~T}$ superconducting MRI scanner produced by German Siemens. The patient was required to lie to scan the head back and forth, and the transverse T1-weighted image (T1WI), T2-weighted image (T2WI), diffusion weighted imaging (DWI), and fluid attenuated inversion recovery (FLAIR) were scanned comprehensively. The specific parameters were as follows: layer thickness was $1.0 \mathrm{~mm}$, layer spacing was $0.5 \mathrm{~mm}$, echo time was $6.6 \mathrm{~ms}$, repetition time was set to $25 \mathrm{~ms}$, scanning sequence was 3D-TOF-MRA, scanning field was $360 \mathrm{~mm} \times 360 \mathrm{~mm}$, and matrix was set to $256 \times 196$.

CT diagnosis and MRI diagnosis of all patients were performed by physicians who were experienced in clinical diagnosis in accordance with the routine operating procedures. The observation indicators included the diagnostic compliance rate (DCR) and diagnosis cost, and inspection time required for various types of CVD was recorded and compared between the two diagnostic methods.

\subsection{Construction of LRMD Algorithm Based on Block Prior.} Related studies showed that the Gaussian mixture model (GMM) can better perform statistical modeling of the noise-free image block prior. The prior information of the noise-free MRI image block was combined with the self-conformity of the MRI image block. The GMM with a prior of noise-free image blocks was adopted to guide the clustering of noisy MRI image blocks to improve the denoising effect of the LRMD algorithm. When a pair of noise MRI $k$ was given, a set of image blocks could be obtained through block division as follows:

$$
M k=\left(M_{1} k, \ldots M_{l} k, \ldots M_{n} k\right)
$$

It was assumed that the GMM parameter collection $\phi$ was known by learning the information of noise-free MRI image blocks, and then $M k$ could be classified into $N$ categories based on the GMM prior $\phi$, representing the matrix composed of all image blocks (vectorization) in the $n^{\text {th }}$ category $(1(n)$ represented the number of the all similar image blocks in the $n^{\text {th }}$ Gaussian category). The structural information included in the same Gaussian category was similar, so $M_{n} k$ could be decomposed as follows:

$$
\bar{M}_{n} k=S_{n}+Y_{n}
$$

In equation (2), $S_{n}$ and $Y_{n}$ refer to the low-rank matrix and the noise matrix, respectively, and the low-rank matrix was the image data after denoising. It was assumed that the noise on each pixel in the image was independent and 
identically distributed, and then equation (3) could be obtained from the perspective of conditional probability:

$$
P\left(\overline{M_{n} k} \mid D_{n}\right) \propto \exp \left(-\frac{1}{\partial^{2}}\left\|M_{n} \overline{k-} D_{n}\right\|_{E}^{2}\right),
$$

where $D_{n}$ could be obtained by minimizing the energy function $Q\left(D_{n}\right)$ in equation (3):

$$
Q\left(D_{n}\right)=\ell\left\|D_{n}\right\|_{*}+\frac{1}{\partial^{2}}\left\|M_{n} \bar{k}-D_{n}\right\|_{E}^{2} .
$$

In equation (4), $\ell$ is a normal number, $\partial$ is the noise standard deviation; $\|\cdot\|_{*}$ refers to the matrix kernel norm, and $\|.\|_{E}^{2}$ represents the Frobenius norm of the matrix. The rank minimization shown in (4) can be optimally solved by minimizing the weighted kernel norm. Let $L \Sigma B^{e}$ be the singular value decomposition (SVD) of $M_{n} k$, then

$$
\widehat{D}_{n}=L S_{i}(\Sigma) B^{e} .
$$

In equation (5), $S_{i}(\Sigma)$ is the singular value contraction operator.

In summary, the given noise MRI Y was combined with the noise-free MRI image block prior (GMM prior) and the MRI image block self-similarity prior to the LRMD model, as shown in the following equation. With this model, the noise-free MRI $X$ can be reconstructed from the noised MRI $Y$ as follows:

$$
\begin{aligned}
\left(\widehat{H}, \widehat{V},\left\{\widehat{D}_{n}\right\}\right)= & \underset{\widehat{H}, \widehat{V},\left\{\widehat{D}_{n}\right\}}{\arg \min } \frac{\beta}{\partial^{2}}\|T-X\|_{2}^{2} \\
& -\log (\mathrm{RI}, A)+\sum_{n=1}^{n} \mathrm{Q}\left(D_{n}\right) .
\end{aligned}
$$

In equation (6), $\beta$ is a normal number and $\partial$ refers to the noise standard deviation. The MRI signal $H$ obtained from (6) was squared, and deviation in (7) was corrected to obtain the finally restored MRI signal $H_{\text {final }}$ as follows:

$$
H_{\text {final }}=\sqrt{\widehat{H}-2 \partial^{2} Q} \text {. }
$$

In equation (7), $Q$ is the identity matrix of $\widehat{H}$.

The DCR of patient could be calculated with the following equation (8):

$$
R=\frac{W}{(K-V)} \times 100 \%
$$

In equation (8), $R$ refers to the DCR, $W$ is the number of patients who meet the diagnosis, $K$ represents the number of discharged patients, and $V$ refers to the number of suspected patients. In the performance analysis of CT diagnosis and MRI diagnosis, accuracy and sensitivity were undertaken as evaluation indicators. The specific equations were as follows:

$$
\begin{gathered}
A=\frac{F_{t}}{T} \times 100 \%, \\
S=\frac{P}{W+F} \times 100 \%,
\end{gathered}
$$

$$
Y=\frac{N}{W+F} \times 100 \%
$$

In equations (9)-(11), $A, S$, and $Y$ refer to the accuracy, sensitivity, and specificity, respectively; $F_{t}$ means the number of cases with accurate prediction; $t$ means the total number of patients; Tmeans the true positive; and $N, F$, and $W$ refer to true negative, false positive, and false negative, respectively.

2.4. Statistical Methods. The data processing was analyzed by SPSS19.0 version statistical software. The measurement data were displayed as mean \pm standard deviation $(\bar{x} \pm s)$, and the count data were shown as percentage (\%). Running time was compared in pairwise with one-way analysis of variance. The comparison of age, height, weight, course of disease, ratio of male to female, and diagnostic accuracy rate among groups was performed by the paired $t$-test. The difference was statistically significant at $P<0.05$.

\section{Results}

3.1. Comparison on Basic Data of Patients. Among the 129 CVD patients who came to our hospital for treatment, 95 were males and 34 were females, aged 10 65 years. The main clinical manifestations were dizziness, headache, decreased vision, and blurred vision. Figure 1 shows the ratio of male to female of patients in general. As shown in Figure 2, there were 30 males and 13 females in the CT group, with an average age of $48 \pm 4.28$ years; there were 33 males and 10 females in the MRI group, aged $49 \pm 3.79$ years in average; and 29 males and 14 females were included in the CT + MRI group with an average age of $47 \pm 5.51$ years.

There were 50 lesions, including 40 single lesions and 3 multiple lesions in CT group, 45 lesions (42 single lesions and 1 multiple lesion) in MRI group, and 44 lesions (41 single lesions and 2 multiple lesions) in CT + MRI group (as illustrated in Figure 3). The lesion was round or irregular in shape. Figures 1-3 reveal that there was no great difference in the pathological conditions and basic data of the patients $(P>0.05)$.

Figure 4 shows the images of a 51-year-old male patient with a history of migraine for 10 years and no history of hypertension. The patient suffered from a sudden generalized tonic-clonic seizure, which lasted about 10 minutes. On admission, the blood pressure was $148 / 80 \mathrm{mmHg}$, there were no traces of trauma except tongue bit and no obvious positive signs of the nervous system. CT plain scan revealed a high-density shadow in the M1 segment of the left middle cerebral artery, with the size of $27 \times 25 \times 26 \mathrm{~mm}$; CT and MRI examinations revealed signs of subarachnoid hemorrhage. 4 days later, the patient was in a coma and was sent to the hospital immediately with dilated pupils. CT scan revealed a large number of intracranial subarachnoid hemorrhage, left temporal lobe hematoma, and rupture into the ventricle; the midline was obviously shifted, forming a temporal lobe hook hernia; and the brainstem showed a low density shadow. The patient died eventually. 


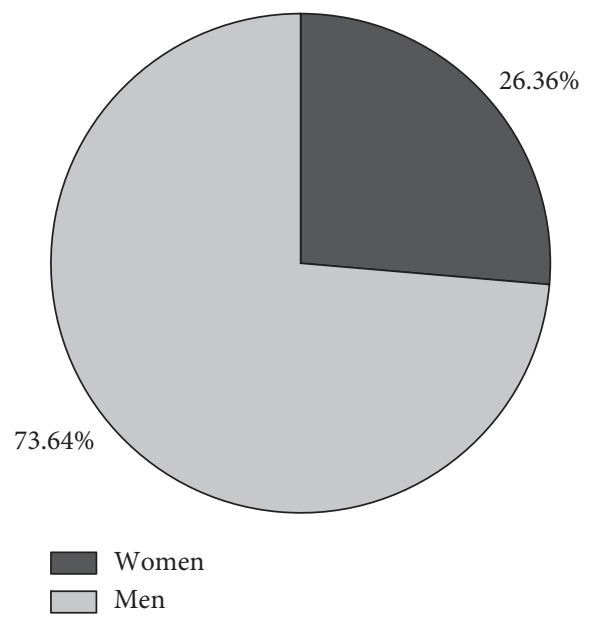

Figure 1: Ratio of male to female of patients.

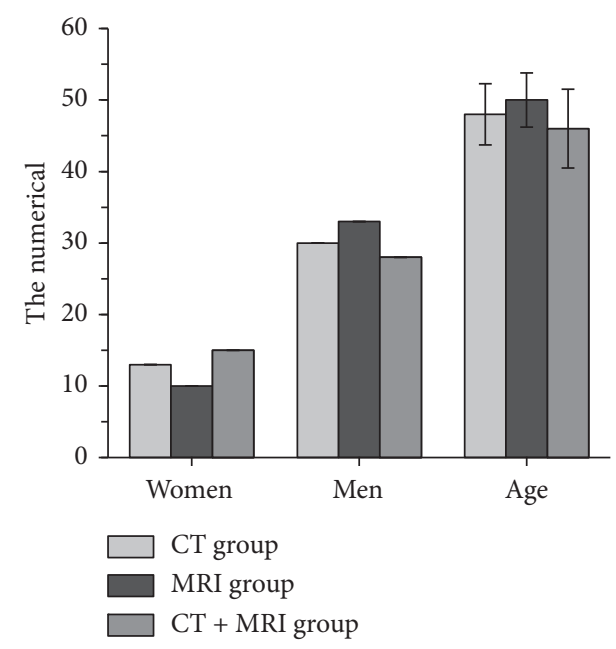

Figure 2: Comparison on basic data of patients.

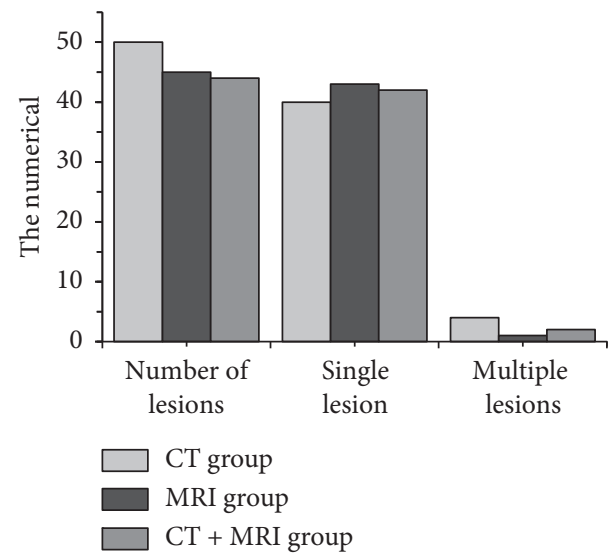

FIGURE 3: Comparison on pathological conditions of patients.

3.2. Comparison on DCR of Patients in Different Groups. As illustrated in Figures 5 and 6, 25 patients with cerebral hemorrhage $(\mathrm{CH}), 10$ patients with cerebral infarction $(\mathrm{CI})$, and 8 patients with CA were diagnosed using the CT diagnosis method. The DCR was 95.1\%, 94.7\%, and 70\%, respectively. 29 patients with $\mathrm{CH}, 7$ patients with $\mathrm{CI}$, and 7 patients with $\mathrm{CA}$ were diagnosed in the MRI group. The DCR was $99.01 \%$, $97.71 \%$, and $100 \%$, respectively. In the $\mathrm{CT}+\mathrm{MRI}$ group, there were 30 cases of $\mathrm{CH}, 10$ cases of $\mathrm{CI}$, and 3 cases of $\mathrm{CA}$, and the DCR was $100 \%, 99.78 \%$, and $100 \%$, respectively. According to the diagnosis results, the positive rates of MRI and CT + MRI for CA were much higher in contrast to the CT, showing statistical differences $(P<0.05)$; MRI diagnosis showed higher DCR than CT but lower DCR than CT + MRI, showing meaningful differences statistically $(P<0.05)$.

\subsection{Comparison on Diagnosis Performance of CT and MRI.} Figure 7 illustrates that the accuracy, sensitivity, and specificity of CT diagnosis were $92.71 \%, 84.94 \%$, and $80.71 \%$, respectively, while those of MRI diagnosis using the LRMD algorithm were $96.28 \%, 88.76 \%$, and $90.62 \%$, respectively. Thus, the accuracy, sensitivity, and specificity of MRI diagnosis under the LRMD algorithm were obviously different from those of CT diagnosis $(P<0.05)$.

3.4. Comparison on CVD Cost of Patients with Different Diagnosis Methods. The DC/C after CT examination was $601.42 \pm 83.61$ yuan, and the TDC was $39,819.2 \pm 198.72$ yuan; the DC/C and TDC of the MRI group were $799.73 \pm 100.02$ yuan and $58,521.67 \pm 301.62$ yuan, respectively. Figures 8 and 9 reveal that the DC/C and TDC in the MRI diagnosis were higher, showing remarkable differences in contrast to those in the CT diagnosis $(P<0.05)$.

3.5. Comparison on Diagnosis Time of Patients with Different Diagnosis Methods. The average diagnosis time in the CT group was $15.97 \pm 3.72$ minutes; and that in the MRI group was $27.18 \pm 5.78$ minutes. Figure 10 indicates that there is a large difference between the two $(P<0.05)$.

\section{Discussion}

CA is a relatively common type of CVD in clinical treatment at present. It has a high incidence, but there is no specific effective treatment plan in clinical practice. Therefore, it is particularly important for early diagnosis and treatment of patients. The block prior-based MRI under the LRMD algorithm can provide sufficient prior information for the image. The priori of the noise-free MRI image block was combined with the nonlocal autophagy prior of the MRI image block, the learning method was adopted, and the GMM was applied to characterize the prior information of the noise-free MRI image block. Next, clustering was performed on the noise MRI image block based on the nonlocal self-phase of the image block to maintain the low rank of the image block matrix, thereby improving the denoising effect of the LRMD model.

The results disclosed that $25 \mathrm{CH}$ patients, $10 \mathrm{CI}$ patients, and $8 \mathrm{CA}$ patients were diagnosed in the CT group, and the DCRs were $95.1 \%, 94.7 \%$, and 70\%, respectively, while $29 \mathrm{CH}$ patients, $7 \mathrm{CI}$ patients, and $7 \mathrm{CA}$ patients were diagnosed in the 


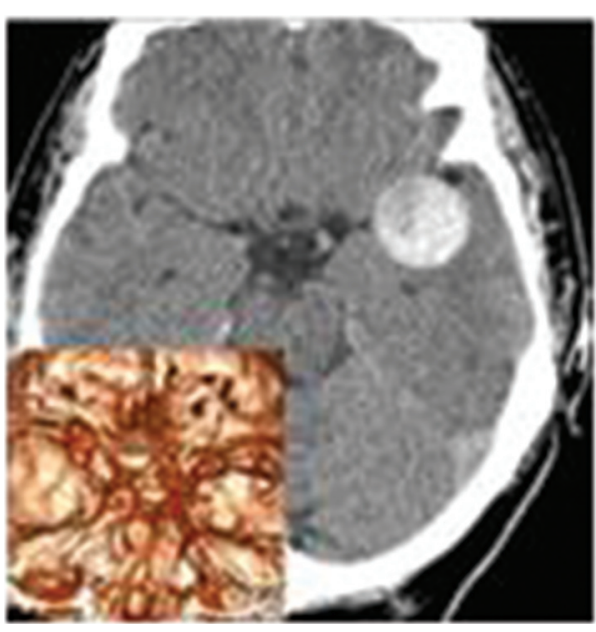

(a)

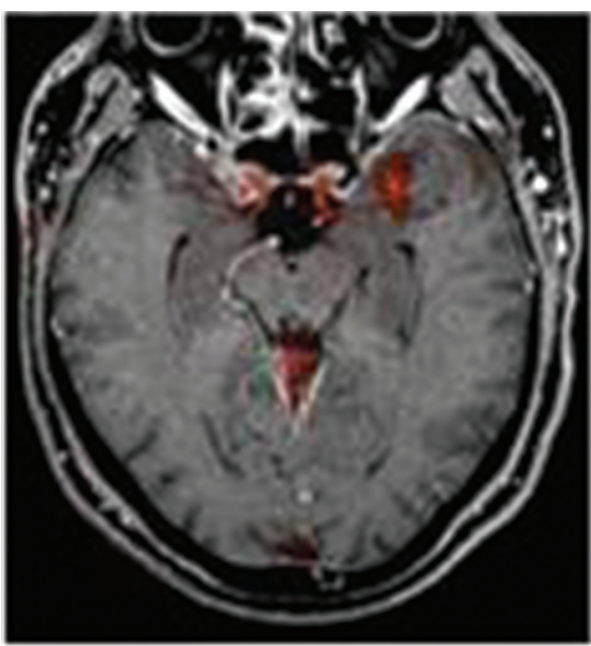

(b)

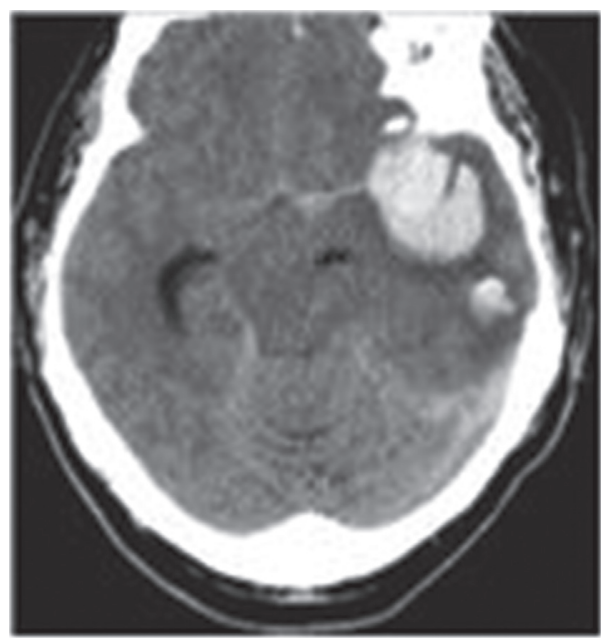

(c)

FIgURE 4: The images of a 51-year-old male patient with a history of migraine for 10 years and no history of hypertension: (a) the visible CA under CT plain scan; (b) sign of subarachnoid hemorrhage in MRI image; and (c) visible intracranial subarachnoid hemorrhage, left temporal lobe hematoma, and rupture into the ventricle under CT plain scan.

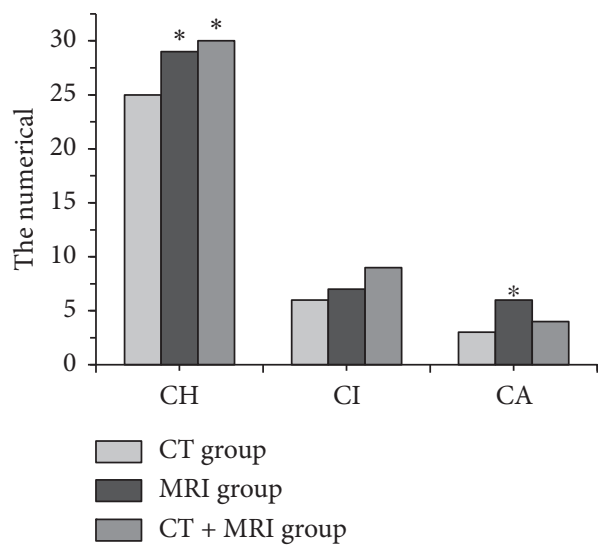

Figure 5: Comparison on diagnosed numbers of patients with different diseases. 


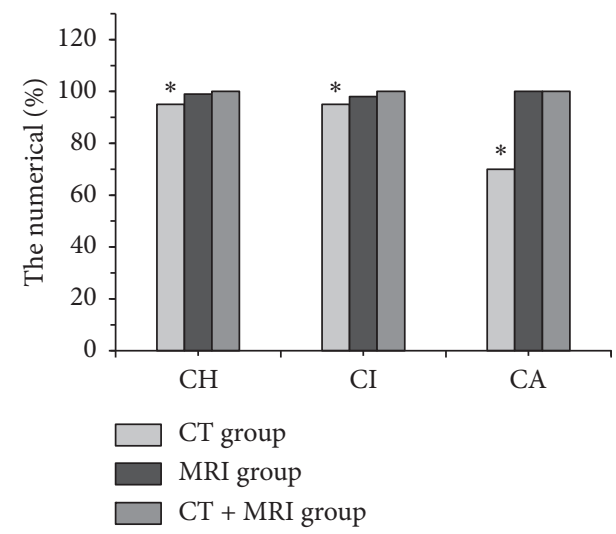

FIgURE 6: Comparison on DCR of three patients with different methods.

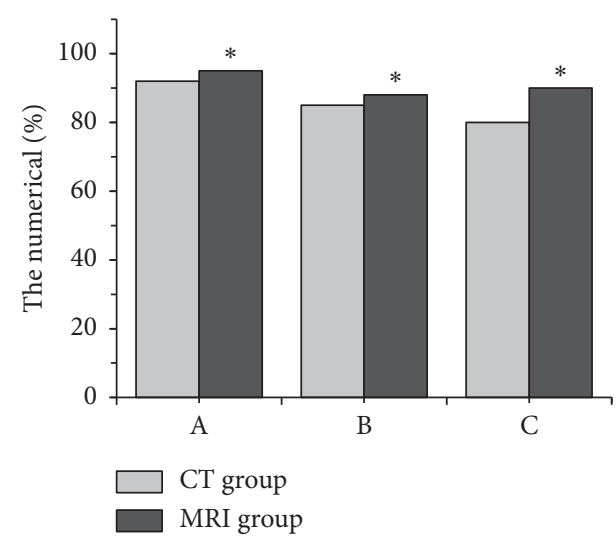

FIGURE 7: Comparison on diagnosis performance of CT and MRI. Note: A, B, and C represent accuracy, sensitivity, and specificity, respectively. ${ }^{*}$ suggests that the difference is statistically obvious $(P<0.05)$.

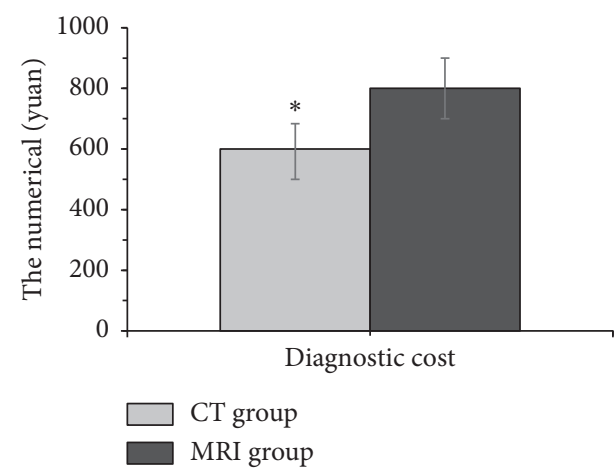

FIGURE 8: DC/C of patient with different diagnosis methods. Note: * suggests that the difference is statistically obvious $(P<0.05)$.

MRI group with the DCRs of $99.01 \%, 97.71 \%$, and $100 \%$, respectively; CT + MRI group was diagnosed with $30 \mathrm{CH}$ cases, 10 CI cases, and 3 CA cases, and the DCRs were $100 \%, 99.78 \%$, and $100 \%$, respectively. According to the experimental results, MRI and CT + MRI diagnosis for CA showed higher positive rates in contrast to the CT diagnosis, with statistical differences $(P<0.05)$; MRI diagnosis showed obviously higher DCR than

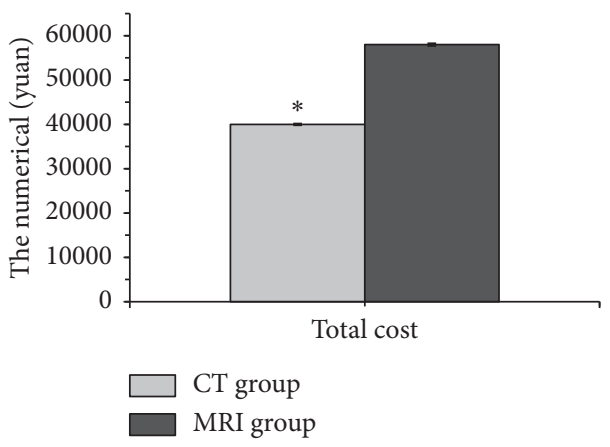

FIgURE 9: TDC of patient with different diagnosis methods. Note: * suggests that the difference is statistically obvious $(P<0.05)$.

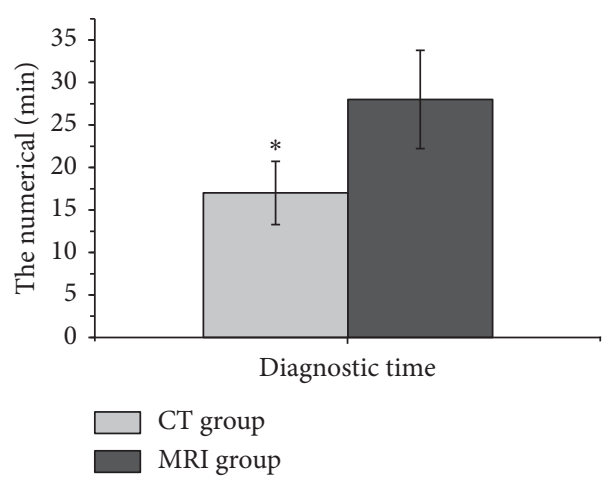

FIGURE 10: Comparison on diagnosis time of patients with different diagnosis methods. Note: ${ }^{*}$ suggests that the difference is statistically obvious $(P<0.05)$.

CT but visibly lower DCR than CT + MRI $(P<0.05)$. This indicated that MRI diagnosis based on the LRMD algorithm had a higher detection rate of CA and had a higher clinical promotion value. Such results were consistent with the research conclusions of Chen et al. [18], which suggested that the algorithm had a good denoising effect. The accuracy, sensitivity, and specificity of CT diagnosis were $92.71 \%, 84.94 \%$, and $80.71 \%$, respectively, while those of MRI diagnosis under the LRMD algorithm were $96.28 \%, 88.76 \%$, and $90.62 \%$, respectively. Thus, the accuracy, sensitivity, and specificity of MRI diagnosis under the LRMD algorithm were dramatically different from those of CT diagnosis $(P<0.05)$; it indicated that the application of the LRMD algorithm in MRI detection can improve the accuracy, sensitivity, and specificity of diagnosis greatly and can quickly detect the CA. Such results were in line with the conclusion of Molyneux et al. [19], providing an important basis for this research theme. The average diagnosis time in the CT group was $15.97 \pm 3.72$ minutes, and that in the MRI group was $27.18 \pm 5.78$ minutes. DC/C and TDC of patient in CT group were $601.42 \pm 83.61$ yuan and $39819.2 \pm 198.72$ yuan, respectively, while those in the MRI group were $799.73 \pm 100.02$ yuan and $58,521.67 \pm 301.62$ yuan. Thus, patients taking MRI diagnosis required more money than patients taking CT diagnosis, which showed that the MRI diagnosis was expensive and time-consumed. Although CT diagnosis was less accurate than MRI diagnosis, the diagnosis time was short and 
the cost was lower, so it was easier for patients to accept. In addition, it was found that the diagnosis rate of CA with $\mathrm{CT}+\mathrm{MRI}$ was higher, and it was worthy of clinical application and promotion.

\section{Conclusion}

The LRMD algorithm was constructed based on block prior theory and then applied to MRI diagnosis of CA patients. It was found that MRI diagnosis under the LRMD algorithm could effectively improve the positive diagnosis rate of CA in CVD patients and had high diagnostic accuracy, sensitivity, and specificity. In summary, both CT and MRI detection had their own advantages for the diagnosis of CA. MRI detection showed a higher diagnosis rate and safety, while CT diagnosis required a short time and less costs, so the two can be diagnosed jointly, which can improve the efficiency and accuracy of clinical diagnosis to a great extent. However, there were still some shortcomings for this study. The number of selected patient samples was too small, leading to a small scope of application of the research results and even some adverse effects on the final results. In follow-up research, it will consider increasing the sample of patients and expand the scope of investigation so as to further explore the new clinical treatment of CA. This study could provide a reliable theoretical basis for the application of $\mathrm{CT}$ and MRI imaging optimization algorithms in diagnosis of CVDs.

\section{Data Availability}

No data were used to support this study.

\section{Conflicts of Interest}

The authors declare that they have no conflicts of interest.

\section{Authors' Contributions}

Daigui Zhang and Lihua Zhou contributed equally to this work.

\section{References}

[1] K. Maeda, R. Motoie, S. Karashima et al., "A case of delayed distal coil migration after coil embolization of an unruptured distal azygos anterior cerebral artery aneurysm: a case report and literature review," Interventional Neuroradiology, vol. 24, no. 6, pp. 643-649, 2018.

[2] A. E. Hussein, D. Brunozzi, S. F. Shakur, R. Ismail, F. T. Charbel, and A. Alaraj, "Cerebral aneurysm size and distal intracranial hemodynamics: an assessment of flow and pulsatility index using quantitative magnetic resonance angiography," Neurosurgery, vol. 83, no. 4, pp. 660-665, 2018.

[3] D. H. Tian, J. Weller, S Hasmat et al., "Adjunct retrograde cerebral perfusion provides superior outcomes compared with hypothermic circulatory arrest alone: a meta-analysis," The Journal of Thoracic and Cardiovascular Surgery, vol. 156, no. 4, pp. 1339-1348, 2018.

[4] J. D. Sokolowski, E. L. Guilliams, M Diaz et al., "Neoplastic cerebral aneurysm from metastatic lung adenocarcinoma with neuroendocrine features," World Neurosurg, vol. 122, pp. 155-160, 2019.
[5] F. Cagnazzo, D. Mantilla, A Rouchaud et al., "Endovascular treatment of very large and giant intracranial aneurysms: comparison between reconstructive and deconstructive techniques-A meta-analysis," American Journal of Neuroradiology, vol. 39, no. 5, pp. 852-858, 2018.

[6] Y. Wang, X. Leng, X. Zhou, W. Li, A. H. Siddiqui, and J. Xiang, "Hemodynamics in a middle cerebral artery aneurysm before its growth and fatal rupture: case study and review of the literature," World Neurosurgery, vol. 119, pp. e395-e402, 2018.

[7] M. Gmeiner, J. Dirnberger, W. Fenz et al., "Virtual cerebral aneurysm clipping with real-time haptic force feedback in neurosurgical education," World Neurosurgery, vol. 112, pp. e313-e323, 2018.

[8] C. S. Gathier, B. D. Van, D. Van et al., "Induced hypertension for delayed cerebral ischemia after aneurysmal subarachnoid hemorrhage: a randomized clinical trial," Stroke, vol. 49, no. 1, pp. 76-83, 2018.

[9] B. L. Hoh, K. Rojas, L Lin et al., "Estrogen deficiency promotes cerebral aneurysm rupture by upregulation of Th17 cells and interleukin-17a which downregulates E-cadherin," Journal of American Heart Association, vol. 7, no. 8, Article ID e008863, 2018.

[10] W. Venderink, M. F. Van, V. J. De, and F. Meijer, "Cerebral aneurysm rebleed with ventricular breakthrough captured by four-dimensional CT angiography," Neuroradiology, vol. 60, no. 7, pp. 665-667, 2018.

[11] M. Katsura, J. Sato, M Akahane et al., "Single-energy metal artifact reduction technique for reducing metallic coil artifacts on post-interventional cerebral CT and CT angiography," Neuroradiology, vol. 60, no. 11, pp. 1141-1150, 2018.

[12] S. D. Hajdu, R. T. Daniel, R. A. Meuli, J. B. Zerlauth, and V. Dunet, "Impact of model-based iterative reconstruction (MBIR) on image quality in cerebral CT angiography before and after intracranial aneurysm treatment," European Journal of Radiology, vol. 102, pp. 109-114, 2018.

[13] D. Schetelig, J. Sedlacik, J. Fiehler et al., "Analysis of the influence of imaging-related uncertainties on cerebral aneurysm deformation quantification using a no-deformation physical flow phantom," Scientific Reports, vol. 8, no. 1, Article ID 11004, 2018.

[14] S. Afat, C. Brockmann, O Nikoubashman et al., "Diagnostic accuracy of simulated low-dose perfusion CT to detect cerebral perfusion impairment after aneurysmal subarachnoid hemorrhage: a retrospective analysis," Radiology, vol. 287, no. 2, pp. 643-650, 2018.

[15] A. K. Petridis, A. Filis, E Chasoglou et al., "Aneurysm wall enhancement in black blood MRI correlates with aneurysm size black blood MRI could serve as an objective criterion of aneurysm stability in near future," Clinical Practice, vol. 8, no. 3, Article ID 1089, 2018.

[16] S. Sindeev, P. G. Arnold, S Frolov et al., "Phase-contrast MRI versus numerical simulation to quantify hemodynamical changes in cerebral aneurysms after flow diverter treatment," Plos One, vol. 13, no. 1, Article ID e0190696, 2018.

[17] Z. Tian, Z. Wang, W Li et al., "Dynamic contrast-enhanced MRI analysis for prognosis of intracranial dissecting aneurysm with intramural haematoma after endovascular treatment: an observational registry study," Stroke and Vascular Neurology, vol. 6, 2020.

[18] X. Chen, Y. Liu, H Tong et al., "Meta-analysis of computed tomography angiography versus magnetic resonance angiography for intracranial aneurysm," Medicine (Baltimore), vol. 97, no. 20, Article ID e10771, 2018. 
[19] A. J. Molyneux, J. Birks, A. Clarke, M. Sneade, and R. S. Kerr, "The durability of endovascular coiling versus neurosurgical clipping of ruptured cerebral aneurysms: 18 year follow-up of the UK cohort of the international subarachnoid aneurysm trial (ISAT)," Lancet, vol. 385, no. 9969, pp. 691-697, 2015. 